\title{
Weed Management in Azuki Bean with Postemergence Herbicides
}

\author{
Nader Soltani*, Lynette Brown, Peter H. Sikkema \\ University of Guelph Ridgetown Campus, Ridgetown, Ontario, Canada \\ Email: *soltanin@uoguelph.ca
}

How to cite this paper: Soltani, N., Brown, L. and Sikkema, P.H. (2020) Weed Management in Azuki Bean with Postemergence Herbicides. American Journal of Plant Sciences, 11, 1467-1477.

https://doi.org/10.4236/ajps.2020.119106

Received: August 7, 2020

Accepted: September 25, 2020

Published: September 28, 2020

Copyright $\odot 2020$ by author(s) and Scientific Research Publishing Inc. This work is licensed under the Creative Commons Attribution International License (CC BY 4.0).

http://creativecommons.org/licenses/by/4.0/ (c) (i) Open Access

\begin{abstract}
A limited number of postemergence (POST) herbicides are available for weed management in azuki bean production in Ontario. A total of three field trials were conducted during 2017 to 2019 at the Huron Research Station, University of Guelph, Exeter, Ontario, Canada to evaluate the efficacy of various postemergence (POST) herbicides for weed control in azuki bean. Fomesafen, acifluorfen and acifluorfen + Assist caused up to $6 \%$ crop injury and weed interference reduced azuki bean yield up to $42 \%$. Bentazon, bentazon Forte, bentazon/acifluorfen, bentazon + fomesafen + Assist ${ }^{\circ}$, bentazon + fomesafen + Turbocharge and bentazon Forte + fomesafen caused up to $16 \%$ injury and weed interference reduced azuki bean yield up to $53 \%$. Fomesafen, acifluorfen, acifluorfen + Assist, bentazon, bentazon Forte, bentazon/acifluorfen, bentazon + fomesafen + Assist, bentazon + fomesafen + Turbocharge ${ }^{\circ}$ and bentazon Forte + fomesafen provided $36 \%$ to $88 \%$ control of redroot pigweed (Amaranthus retroflexus L.), common ragweed (Ambrosia artemesiifolia L.), and common lambsquarters (Chenopodium album L.). Fomesafen, acifluorfen + Assist and acifluorfen provided $59 \%$ to $83 \%$ control of flower-of-an-hour (Hibiscus trionum L.), but bentazon, bentazon Forte, bentazon/acifluorfen, bentazon + fomesafen + Assist bentazon + fomesafen + Turbocharge $e^{\circ}$ and bentazon Forte + fomesafen provided $78 \%$ to $99 \%$ control of $H$. trionum. All POST herbicides evaluated provide $99 \%$ to $100 \%$ control of wild mustard (Sinapis arvensis L.) in azuki bean. Based on these results, none of the POST herbicide evaluated that included bentazon can be used safely for the control of annual broadleaved weeds in azuki bean under Ontario environmental conditions.
\end{abstract}

\section{Keywords}

Annual Weeds, Density, Dry Weight, Maturity, Seed Yield, 
Phaseolus vulgaris, Vigna angularis

\section{Introduction}

Dry bean production is important to the economy and agriculture in Canada. Dry bean growers in Canada produced 249,000, 322,000, 341,000 and 317,000 tonnes of dry bean in 2016, 2017, 2018 and 2019, respectively [1]. Ontario farmers produce most of the dry beans grown in Canada. In 2019, dry bean growers in Ontario planted 53,000 hectares of dry bean including white, black, cranberry, kidney, azuki and other market classes with a total farm gate value of approximately \$100,000,000 [2]. Azuki bean [Vigna angularis (Willd.) Ohwi \& Ohashi] is a specialty market class of dry bean grown mostly for the export market to Asia where it is used in confectionery products [3]. Azuki bean thrives well under Ontario environmental conditions and has become popular among dry bean growers in Ontario. There was an increase of $46 \%$ in azuki production in 2019 compared to 2018 [2]. One of the largest production obstacles in azuki bean production is yield loss from weed interference [4]. The Weed Science Society of America (WSSA) reported that dry bean yield was reduced $71 \%$ compared to $50 \%$ in corn and $52 \%$ in soybean due to weed competition [5] [6] [7]. Azuki bean growers need new herbicide options to control problematic weeds in their production.

Fomesafen is a diphenyl ether herbicide that causes cell membrane destruction in susceptible plants [8]. Fomesafen can control/suppress problematic broadleaved weeds in Ontario including redroot pigweed (Amaranthus retroflexus L.), common ragweed (Ambrosia artemesiifolia L.), wild mustard (Sinapis arvensis L.), ladysthumb (Polygonum persicaria L.), and eastern black nightshade (Solanum ptycanthum Dun. ex DC. pp.) [9]. Fomesfen is primarily applied postemergence (POST) although it has some short residual activity against some broadleaved weeds [8].

Acifluorfen is another diphenyl ether POST herbicide that controls broadleaved weeds such as jimsonweed (Datura stramonium L.), P. persicaria, S. arvensis, A. retroflexus, $A$. artemesiifolia and $S$. ptycanthum [9]. Unlike fomesafen, acifluorfen has minimal to no residual activity against weeds [8].

Bentazon is a benzothiadiazole POST herbicide that inhibits photosynthesis at the photosystem II (PS II) in susceptible plants [9]. Bentazon can control key broadleaved weeds including common lambsquarters (Chenopodium album L.), P. persicaria, S. arvensis, A. theophrasti, X. strumarium, jimsonweed (Datura stramonium L.), purslane (Portulaca oleracea L.), wild radish (Raphanus raphanistrum L.), flower-of-an-hour (Hibiscus trionum L.), stinkweed (Thlaspi arvense L.), shepherdspurse (Capsella bursa-pastoris (L.) Medic) and common chickweed (Stellaria media (L.) Vill.) including group 2 and 5 resistant biotypes [8] [9]. Similar to acifluorfen, bentazon is applied POST and is absorbed by foliage (not translocated). It has no residual activity against weeds, therefore weeds 
emerged after application will not be controlled [9].

Fomesafen, acifluorfen and bentazon are often used in combination with an adjuvant to improve weed control efficacy and provide a greater degree of weed control consistency under varying environments [8]. Assist is a mineral oil/surfactant adjuvant that consists of $83 \%$ paraffin base mineral oil plus $17 \%$ surfactant blend that is often added to fomesafen or acifluorfen [8]. Turbocharge is a surfactant/solvent adjuvant that consists of a $39.5 \%$ surfactant blend plus $50 \%$ solvent (mineral oil) and is often added to fomesafen [8]. Bentazon Forte does not require additional adjuvants [8].

Currently, fomesafen is the only POST herbicide registered for the control of broadleaved weeds in azuki bean production in Ontario [8]. Fomesafen does not adequately control common weeds in Ontario such as $C$. album, giant ragweed (Ambrosia trifida L.), P. persicaria and A. theophrasti [8]. The co-application of fomesafen with bentazon and bentazon with acifluorfen using different adjuvants available can be new herbicide options for weed management in azuki bean production.

There is little knowledge on the crop safety and effectiveness of fomesafen, acifluorfen and bentazon (with or without adjuvants), applied POST alone and in combination for weed control in azuki bean under Ontario environmental conditions. The co-application of these herbicides has the potential to produce efficacious full-season control of troublesome weeds in azuki bean in Ontario.

The purpose of this study was to determine the crop safety and effectiveness of fomesafen, acifluorfen, acifluorfen + Assist, bentazon, bentazon Forte, bentazon/acifluorfen, bentazon + fomesafen + Assist', bentazon + fomesafen + Turbocharge and bentazon Forte + fomesafen to control common weeds in azuki bean production.

\section{Materials and Methods}

\subsection{Experimental Methods}

Field experiments were established in late May to early June of 2017, 2018 and 2019 at the Huron Research Station, University of Guelph, Exeter, Ontario, Canada with a five-crop rotation of winter wheat-corn-soybean-oats-azuki bean.

Each experiment was arranged in a randomized complete block design with 4 replicates. Treatments evaluated are listed in Table 1 . The experimental plots were $3.0 \mathrm{~m}$ wide and $10.0 \mathrm{~m}$ long. Azuki bean "Erimo" was seeded $4 \mathrm{~cm}$ deep at the rate of approximately 200,000 seeds $\mathrm{ha}^{-1}$ in rows that were $75 \mathrm{~cm}$ apart in late May to early June.

Herbicide treatments were applied postemergence 3 - 4 weeks after seeding when azuki beans were at the 1 - 2 trifoliate leaf stage. Herbicides were applied with a $\mathrm{CO}_{2}$-pressurized backpack sprayer calibrated to deliver $200 \mathrm{~L} \cdot \mathrm{ha}^{-1}$ at 240 $\mathrm{kPa}$. The spray boom was $1.5 \mathrm{~m}$ long equipped with 4 ultra-low drift (ULD 120-02, Pentair-Hypro, New Brighton, Minnesota) nozzles spaced $0.5 \mathrm{~m}$ apart, producing a spray width of $2.0 \mathrm{~m}$. 
Table 1. Visible injury 2 and 4 WAT, percent moisture at maturity and yield of adzuki bean after various herbicides were applied POST at Exeter, ON, Canada (2017-2019). Means followed by a different letter within a column are significantly different according to a Tukey-Kramer multiple range test at $\mathrm{P}<0.05$. $^{\mathrm{a}}$

\begin{tabular}{|c|c|c|c|c|c|}
\hline \multirow[t]{2}{*}{ Treatment } & \multirow{2}{*}{$\frac{\text { Rate }}{\left(\mathrm{g} \mathrm{ai} \mathrm{ha}^{-1}\right)}$} & \multicolumn{2}{|c|}{ Injury (\%) } & \multirow{2}{*}{$\begin{array}{c}\text { Moisture } \\
(\%)\end{array}$} & \multirow{2}{*}{$\begin{array}{c}\text { Yield } \\
\left(\mathrm{t} \cdot \mathrm{ha}^{-1}\right)\end{array}$} \\
\hline & & $2 \mathrm{WAT}$ & $4 \mathrm{WAT}$ & & \\
\hline Weed-free control & & 0.0 & 0.0 & $14.5 \mathrm{a}$ & $1.9 \mathrm{a}$ \\
\hline Weedy control & & $0.0 \mathrm{a}$ & $0.0 \mathrm{a}$ & $15.7 \mathrm{c}$ & $0.5 \mathrm{~d}$ \\
\hline Fomesafen $^{\mathrm{b}}$ & 240 & $4.4 \mathrm{~b}$ & $0.7 \mathrm{ab}$ & $15.0 \mathrm{ab}$ & $1.1 \mathrm{bc}$ \\
\hline Acifluorfen $^{c}$ & 300 & $6.1 \mathrm{~b}$ & $2.3 \mathrm{abc}$ & $15.0 \mathrm{ab}$ & $1.2 \mathrm{~b}$ \\
\hline Acifluorfen & 600 & $6.3 \mathrm{~b}$ & $2.6 \mathrm{abc}$ & $14.9 \mathrm{ab}$ & $1.1 \mathrm{bc}$ \\
\hline Bentazon $^{c}$ & 1080 & $16.4 \mathrm{c}$ & $6.3 c$ & $15.7 \mathrm{c}$ & $0.9 \mathrm{c}$ \\
\hline Bentazon Forte & 1080 & $13.4 \mathrm{c}$ & $5.8 \mathrm{c}$ & $15.8 \mathrm{c}$ & $1.0 \mathrm{bc}$ \\
\hline Bentazon/acifluorfen ${ }^{\mathrm{d}}$ & 840 & $14.1 \mathrm{c}$ & $5.3 \mathrm{c}$ & $15.3 \mathrm{bc}$ & $1.0 \mathrm{bc}$ \\
\hline Bentazon + fomesafen ${ }^{e}$ & $840+140$ & $15.8 \mathrm{c}$ & $5.3 \mathrm{c}$ & $15.6 \mathrm{bc}$ & $1.1 \mathrm{bc}$ \\
\hline Bentazon + fomesafen ${ }^{f}$ & $840+140$ & $14.8 \mathrm{c}$ & $4.9 \mathrm{bc}$ & $15.5 \mathrm{bc}$ & $1.2 \mathrm{~b}$ \\
\hline Bentazon Forte + fomesafen & $1080+140$ & $15.9 \mathrm{c}$ & $5.1 \mathrm{c}$ & $15.7 \mathrm{c}$ & $1.1 \mathrm{bc}$ \\
\hline
\end{tabular}

${ }^{a} A$ Abbreviations: POST, postemergence; WAT, weeks after herbicide treatment; Includes Turbocharge at $0.5 \%$ $\mathrm{v} / \mathrm{v}$; Includes Assist at $0.5 \% \mathrm{v} / \mathrm{v}$; ${ }^{\mathrm{d}}$ Includes Assist at $1.5 \mathrm{l} \cdot \mathrm{ha}^{-1}$; ' Includes Assist at $2.0 \mathrm{l} \cdot \mathrm{ha}^{-1}$; ${ }^{\mathrm{f}}$ Includes Turbocharge at $0.5 \mathrm{l} \cdot \mathrm{ha}^{-1}$.

Azuki bean visible injury was evaluated 2 and 4 weeks after herbicide application (WAT) and weed control were assessed 4 and 8 WAT on a scale of 0 (no injury/control) to $100 \%$ (total plant necrosis/weed control). Weed density and aboveground dry weight (biomass) were measured 8 WAT from two $0.25 \mathrm{~m}^{2}$ quadrats placed between the centre two rows from each plot. Azuki bean seed yield (adjusted to 13\% moisture) was determined by harvesting the middle two rows of each plot at maturity.

\subsection{Statistical Analyses}

The experimental design was a randomized complete block with 4 replications. Data were analyzed using the GLIMMIX procedure in SAS [10]. The fixed effect was herbicide treatment and random effects were year-location combinations (environment), replicate within environment and the environment by treatment interaction. The Shapiro-Wilk statistic, fit statistics, residual plots and the potential distributions were used to identify the best distribution and associated link function for each parameter. Least square means (LSMEANS) were calculated on the data scale by using the inverse link function, and pairwise comparisons were subjected to Tukey's adjustment before determining treatment differences at $\mathrm{P}<$ 0.05 . The normal distribution and identity link were used for adzuki bean injury $4 \mathrm{WAT}$, percent visible weed control at 4 and $8 \mathrm{WAT}$ for redroot pigweed, ragweed and common lambsquarters and percent adzuki bean moisture and yield at harvest. The arcsine square root distribution and identity link were used for 
percent visible adzuki bean injury 2 WAT as well as percent visible weed control at 4 and 8 WAT for flower-of-an-hour and wild mustard. Weed density and dry biomass were analyzed using the lognormal distribution and identity link. The weedy control was assigned a value of 0 for injury and weed control, and the weed-free control was assigned a value of 0 for injury, weed density and biomass, or 100 for weed control and was excluded from the analysis due to zero variance. Comparisons were still possible between the other treatments and the value zero using the LSMEANS output and differences were identified. Arcsine square root and lognormal distributions were back-transformed for the presentation of results.

\section{Results and Discussion}

\subsection{Crop Injury}

At 2 and 4 WAE, fomesafen, acifluorfen (with Assist'), acifluorfen, bentazon, bentazon Forte, bentazon/acifluorfen, bentazon + fomesafen (with Assist'), bentazon + fomesafen (with Turbocharge ${ }^{\circ}$ ) and bentazon Forte + fomesafen caused as much as $4 \%, 6 \%, 6 \%, 16 \%, 13 \%, 14 \%, 16 \%, 15 \%$ and $16 \%$ injury in azuki bean, respectively (Table 1 ). Azuki bean seed moisture content was $1.2 \%$ higher in the weedy control indicating delayed maturity if weeds are not controlled. Fomesafen and acifluorfen (with/without Assist ${ }^{\circ}$ ) did not affect maturity but bentazon, bentazon Forte, bentazon/acifluorfen, bentazon + fomesafen (with Assist ${ }^{\circ}$ ), bentazon + fomesafen (with Turbocharge ${ }^{\circ}$ ) and bentazon Forte + fomesafen delayed maturity of azuki bean (Table 1 ). Weed interference reduced azuki bean yield $74 \%$ compared to the weed-free control. Additionally, weed interference in plots treated with fomesafen, acifluorfen (with Assist'), acifluorfen, bentazon, bentazon Forte, bentazon/acifluorfen, bentazon + fomesafen (with Assist ${ }^{\circ}$ ), bentazon + fomesafen (with Turbocharge ${ }^{\circ}$ ) and bentazon Forte + fomesafen reduced azuki bean yield 42\%, 37\%, 42\%, 53\%, 47\%, 47\%, 42\%, 37\% and 42\%, respectively (Table 1). Results are similar to other studies in which fomesafen, acifluorfen and bentazon caused as much as $18 \%, 20 \%$ and $28 \%$ injury in azuki bean, respectively [11]. Another study found $5 \%$ to $20 \%, 4 \%$ to $18 \%$ and $19 \%$ to $28 \%$ injury in azuki bean with acifluorfen, fomesafen and bentazon applied POST in azuki bean, respectively [12]. Other studies have reported 14 to $28 \%$ injury with no effect on seed yield in azuki bean with fomesafen applied POST in azuki bean [13]. In contrast, other studies have shown less than $5 \%$ injury with bentazon (Forte) at $840 \mathrm{~g}$ ai ha ${ }^{-1}$, fomesafen at $240 \mathrm{~g}$ ai ha $\mathrm{h}^{-1}$ and bentazon Forte + fomesafen at $840+240 \mathrm{~g}$ ai ha $^{-1}$ applied POST. in the Phaseolus market classes of dry beans [14].

\subsection{Weed Control}

Weeds selected for analysis needed to be present in at least 2 out of the 3 environments and included $A$. retroflexus (2/3), A. artemesiifolia (2/3), C. album $(3 / 3), H$. trionum (3/3) and $S$. arvensis $(2 / 3)$. 


\subsubsection{Fomesafen}

At 4 and 8 WAT, fomesafen applied POST at $240 \mathrm{~g}^{\text {ai }} \mathrm{ha}^{-1}$ controlled $A$. retroflexus $59 \%$ to $79 \%$, $A$. artemesiifolia $74 \%$ to $83 \%, C$. album $45 \%$ to $58 \%, H$. trionum $63 \%$ to $77 \%$, and $S$. arvensis $100 \%$ (Tables 2-6). Fomesafen applied POST at $240 \mathrm{~g}$ ai ha ${ }^{-1}$ did not reduce density or dry weight of weeds evaluated except for the dry weight of $A$. artemesiifolia which was reduced $92 \%$ and density and dry weight of $S$. arvensis which was reduced $100 \%$ compared to the weedy control (Tables 2-6). In other studies, Bailey et al. [15] reported 99\% control of $A$. artemesiifolia and $90 \%$ control of $C$. album with fomesafen applied POST at $70 \mathrm{~g}$ ai ha ${ }^{-1}$. Wilson [16] reported that fomesafen applied POST at $280 \mathrm{~g}$ ai $\mathrm{ha}^{-1}$ controlled $A$. retroflexus $94 \%$ and $C$. album $71 \%$. In other studies, fomesafen applied POST at $240 \mathrm{~g}_{\text {ai }} \mathrm{ha}^{-1}$ controlled A. retroflexus $85 \%$ to $86 \%$, A. artemesiifolia $90 \%$ to $93 \%$ and C. album 53\% to $60 \%$ [14].

\subsubsection{Acifluorfen}

At 4 and 8 WAT, acifluorfen ( $300 \mathrm{~g}$ ai ha ${ }^{-1}$ applied with Assist ${ }^{\circ}$ ) or acifluorfen (600 g ai ha ${ }^{-1}$ ) applied POST controlled A. retroflexus $77 \%$ to $88 \%, A$. artemesiifolia $73 \%$ to $77 \%, C$. album $38 \%$ to $57 \%, H$. trionum $59 \%$ to $83 \%$, and $S$. arvensis $99 \%$ to $100 \%$ (Tables 2-6). Acifluorfen (300 $\mathrm{g}$ ai ha ${ }^{-1}$ applied with Assist ${ }^{\circ}$ ) or acifluorfen $\left(600 \mathrm{~g}_{\text {ai }} \mathrm{ha}^{-1}\right)$ did not reduce density or dry weight of $A$. retroflexus, $A$. artemesiifolia, $C$. album and $H$. trionum, but decreased density and biomass of $S$. arvensis $100 \%$ compared to the weedy control (Tables 2-6).

Table 2. Percent visible control 4 and 8 WAT, density and dry weight of redroot pigweed after various herbicides were applied POST at Exeter, ON, Canada (2017-2019). Means followed by a different letter within a column are significantly different according to a Tukey-Kramer multiple range test at $\mathrm{P}<0.05$.

\begin{tabular}{|c|c|c|c|c|c|}
\hline \multirow[t]{2}{*}{ Treatment } & \multicolumn{3}{|c|}{ Control (\%) } & \multirow[b]{2}{*}{$\begin{array}{c}\text { Density } \\
\left(\text { no. } \mathrm{m}^{-2}\right)\end{array}$} & \multirow[b]{2}{*}{$\begin{array}{c}\text { Dry weight } \\
\left(\mathrm{g} \cdot \mathrm{m}^{-2}\right)\end{array}$} \\
\hline & $\left(\mathrm{g} \mathrm{ai} \mathrm{ha}^{-1}\right)$ & $4 \mathrm{WAT}$ & $8 \mathrm{WAT}$ & & \\
\hline Weed-free control & & 100 & 100 & $0.0 \mathrm{a}$ & $0.0 \mathrm{a}$ \\
\hline Weedy control & & $0 \mathrm{~d}$ & $0 \mathrm{~d}$ & $13.1 \mathrm{c}$ & $46.0 \mathrm{c}$ \\
\hline Fomesafen $^{\mathrm{b}}$ & 240 & $79 \mathrm{ab}$ & $59 \mathrm{abc}$ & $7.1 \mathrm{bc}$ & $7.7 \mathrm{bc}$ \\
\hline Acifluorfen $^{c}$ & 300 & $88 \mathrm{a}$ & $77 \mathrm{ab}$ & $4.9 \mathrm{~b}$ & $0.9 \mathrm{ab}$ \\
\hline Acifluorfen & 600 & $88 \mathrm{a}$ & $79 \mathrm{a}$ & $4.9 \mathrm{~b}$ & $3.4 \mathrm{bc}$ \\
\hline Bentazon $^{c}$ & 1080 & $64 \mathrm{c}$ & $47 \mathrm{abc}$ & $6.2 \mathrm{~b}$ & $34.0 \mathrm{bc}$ \\
\hline Bentazon Forte & 1080 & $58 \mathrm{c}$ & $46 \mathrm{abc}$ & $7.7 \mathrm{bc}$ & $30.1 \mathrm{bc}$ \\
\hline Bentazon/acifluorfen $^{\mathrm{d}}$ & 840 & $67 \mathrm{bc}$ & $46 \mathrm{abc}$ & $6.7 \mathrm{bc}$ & $26.3 \mathrm{bc}$ \\
\hline Bentazon + fomesafen ${ }^{e}$ & $840+140$ & $65 c$ & $43 \mathrm{bc}$ & $7.4 \mathrm{bc}$ & $19.5 \mathrm{bc}$ \\
\hline Bentazon + fomesafen ${ }^{\mathrm{f}}$ & $840+140$ & $70 \mathrm{bc}$ & $46 \mathrm{abc}$ & $6.0 \mathrm{~b}$ & $21.1 \mathrm{bc}$ \\
\hline Bentazon Forte + fomesafen & $1080+140$ & $68 \mathrm{bc}$ & $36 \mathrm{c}$ & $8.5 \mathrm{bc}$ & $28.0 \mathrm{bc}$ \\
\hline
\end{tabular}

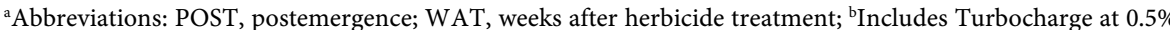
v/v; ' Includes Assist at $0.5 \% \mathrm{v} / \mathrm{v}$; ${ }^{\mathrm{d}}$ Includes Assist at $1.5 \mathrm{l} \cdot \mathrm{ha}^{-1}$; ${ }^{\mathrm{e}}$ Includes Assist at $2.0 \mathrm{l} \cdot \mathrm{ha}^{-1}$; $^{\mathrm{f}}$ Includes Turbocharge at $0.51 \cdot \mathrm{ha}^{-1}$. 
Table 3. Percent visible control 4 and 8 WAT, density and dry weight of common ragweed after various herbicides were applied POST at Exeter, ON, Canada (2017-2019). Means followed by a different letter within a column are significantly different according to a Tukey-Kramer multiple range test at $\mathrm{P}<0.05$. $^{\mathrm{a}}$

\begin{tabular}{|c|c|c|c|c|c|}
\hline \multirow[t]{2}{*}{ Treatment } & \multirow{2}{*}{$\frac{\text { Rate }}{\left(\mathrm{g}_{\mathrm{ai} \mathrm{ha}}{ }^{-1}\right)}$} & \multicolumn{2}{|c|}{ Control (\%) } & \multirow[b]{2}{*}{$\begin{array}{c}\text { Density } \\
\left(\text { no. } \mathrm{m}^{-2}\right)\end{array}$} & \multirow[b]{2}{*}{$\begin{array}{c}\text { Dry weight } \\
\left(\mathrm{g} \cdot \mathrm{m}^{-2}\right)\end{array}$} \\
\hline & & $4 \mathrm{WAT}$ & $8 \mathrm{WAT}$ & & \\
\hline Weed-free control & & 100 & 100 & $0.0 \mathrm{a}$ & $0.0 \mathrm{a}$ \\
\hline Weedy control & & $0 \mathrm{~d}$ & $0 \mathrm{c}$ & $10.7 \mathrm{~b}$ & $53.9 \mathrm{c}$ \\
\hline Fomesafen ${ }^{\mathrm{b}}$ & 240 & $83 \mathrm{a}$ & $74 \mathrm{ab}$ & $6.1 \mathrm{ab}$ & $4.4 \mathrm{ab}$ \\
\hline Acifluorfen $^{c}$ & 300 & $77 \mathrm{ab}$ & $76 \mathrm{a}$ & $7.5 \mathrm{~b}$ & $5.2 \mathrm{abc}$ \\
\hline Acifluorfen & 600 & $76 \mathrm{ab}$ & $73 \mathrm{ab}$ & $8.8 \mathrm{~b}$ & $8.9 \mathrm{abc}$ \\
\hline Bentazon $^{c}$ & 1080 & $51 \mathrm{c}$ & $53 \mathrm{ab}$ & $10.1 \mathrm{~b}$ & $30.3 \mathrm{bc}$ \\
\hline Bentazon Forte & 1080 & $61 \mathrm{bc}$ & $51 \mathrm{~b}$ & $9.3 \mathrm{~b}$ & $46.6 \mathrm{bc}$ \\
\hline Bentazon/acifluorfen ${ }^{\mathrm{d}}$ & 840 & $66 \mathrm{bc}$ & $59 \mathrm{ab}$ & $10.7 \mathrm{~b}$ & $23.3 \mathrm{bc}$ \\
\hline Bentazon + fomesafen ${ }^{e}$ & $840+140$ & $66 \mathrm{bc}$ & $63 \mathrm{ab}$ & $6.7 \mathrm{ab}$ & $16.9 \mathrm{abc}$ \\
\hline Bentazon + fomesafen ${ }^{f}$ & $840+140$ & $68 \mathrm{ab}$ & $72 \mathrm{ab}$ & $8.4 \mathrm{~b}$ & $20.9 \mathrm{bc}$ \\
\hline Bentazon Forte + fomesafen & $1080+140$ & $70 \mathrm{ab}$ & $69 \mathrm{ab}$ & $7.9 \mathrm{~b}$ & $24.9 \mathrm{bc}$ \\
\hline
\end{tabular}

${ }^{a}$ Abbreviations: POST, postemergence; WAT, weeks after herbicide treatment; 'Includes Turbocharge at $0.5 \%$

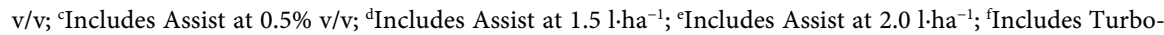
charge at $0.51 \cdot \mathrm{ha}^{-1}$.

Table 4. Percent visible control 4 and 8 WAT, density and dry weight of lambsquarters after various herbicides were applied POST at Exeter, ON, Canada (2017-2019). Means followed by a different letter within a column are significantly different according to a Tukey-Kramer multiple range test at $\mathrm{P}<0.05$. $^{\mathrm{a}}$

\begin{tabular}{|c|c|c|c|c|c|}
\hline \multirow[t]{2}{*}{ Treatment } & \multirow{2}{*}{$\frac{\text { Rate }}{\left(\mathrm{g} \mathrm{ai} \mathrm{ha}^{-1}\right)}$} & \multicolumn{2}{|c|}{ Control (\%) } & \multirow[b]{2}{*}{$\begin{array}{c}\text { Density } \\
\left(\text { no. } \mathrm{m}^{-2}\right)\end{array}$} & \multirow[b]{2}{*}{$\begin{array}{c}\text { Dry weight } \\
\left(\mathrm{g} \cdot \mathrm{m}^{-2}\right)\end{array}$} \\
\hline & & $4 \mathrm{WAT}$ & $8 \mathrm{WAT}$ & & \\
\hline Weed-free control & & 100 & 100 & $0.0 \mathrm{a}$ & $0.0 \mathrm{a}$ \\
\hline Weedy control & & $0 \mathrm{e}$ & $0 \mathrm{~g}$ & $25.3 \mathrm{~d}$ & $24.0 \mathrm{de}$ \\
\hline Fomesafen $^{\mathrm{b}}$ & 240 & $58 \mathrm{~cd}$ & $45 \mathrm{def}$ & $18.7 \mathrm{bcd}$ & $21.2 \mathrm{cde}$ \\
\hline Acifluorfen $^{c}$ & 300 & $57 \mathrm{~cd}$ & $38 \mathrm{f}$ & $22.1 \mathrm{~cd}$ & $29.8 \mathrm{cde}$ \\
\hline Acifluorfen & 600 & $53 \mathrm{~d}$ & $41 \mathrm{ef}$ & $25.4 \mathrm{~d}$ & $31.9 \mathrm{e}$ \\
\hline Bentazon $^{c}$ & 1080 & $66 \mathrm{bcd}$ & 55 bcde & $13.3 \mathrm{bcd}$ & 12.9 bcde \\
\hline Bentazon Forte & 1080 & $87 \mathrm{a}$ & $78 \mathrm{a}$ & $8.4 \mathrm{~b}$ & $8.2 \mathrm{~b}$ \\
\hline Bentazon/acifluorfen ${ }^{\mathrm{d}}$ & 840 & $72 \mathrm{abc}$ & $59 \mathrm{bcd}$ & $13.9 \mathrm{bcd}$ & 15.5 bcde \\
\hline Bentazon + fomesafen ${ }^{e}$ & $840+140$ & $61 \mathrm{~cd}$ & $52 \mathrm{cdef}$ & $14.2 \mathrm{bcd}$ & $20.0 \mathrm{cde}$ \\
\hline Bentazon + fomesafen ${ }^{f}$ & $840+140$ & $83 \mathrm{a}$ & $72 \mathrm{ab}$ & $8.1 \mathrm{~b}$ & $10.6 \mathrm{bcd}$ \\
\hline Bentazon Forte + fomesafen & $1080+140$ & $82 \mathrm{ab}$ & $65 \mathrm{abc}$ & $9.1 b c$ & $8.9 b c$ \\
\hline
\end{tabular}

${ }^{a}$ Abbreviations: POST, postemergence; WAT, weeks after herbicide treatment; ${ }^{b}$ Includes Turbocharge at $0.5 \%$

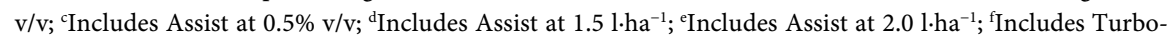
charge at $0.5 \mathrm{l} \cdot \mathrm{ha}^{-1}$. 
Table 5. Percent visible control 4 and 8 WAT, density and dry weight of flower-of-an-hour after various herbicides were applied POST at Exeter, ON, Canada (2017-2019). Means followed by a different letter within a column are significantly different according to a Tukey-Kramer multiple range test at $\mathrm{P}<0.05$. $^{\mathrm{a}}$

\begin{tabular}{|c|c|c|c|c|c|}
\hline \multirow[t]{2}{*}{ Treatment } & \multirow{2}{*}{$\frac{\text { Rate }}{\left(\mathrm{g}_{\mathrm{ai} \mathrm{ha}}{ }^{-1}\right)}$} & \multicolumn{2}{|c|}{ Control (\%) } & \multirow[b]{2}{*}{$\begin{array}{c}\text { Density } \\
\left(\text { no. } \mathrm{m}^{-2}\right)\end{array}$} & \multirow[b]{2}{*}{$\begin{array}{l}\text { Dry weight } \\
\left(\mathrm{g} \cdot \mathrm{m}^{-2}\right)\end{array}$} \\
\hline & & $4 \mathrm{WAT}$ & 8 WAT & & \\
\hline Weed-free control & & 100 & 100 & $0.0 \mathrm{a}$ & $0.0 \mathrm{a}$ \\
\hline Weedy control & & $0 \mathrm{~d}$ & $0 \mathrm{~d}$ & $13.54 \mathrm{~d}$ & $8.35 \mathrm{e}$ \\
\hline Fomesafen $^{\mathrm{b}}$ & 240 & $77 \mathrm{bc}$ & $63 \mathrm{bc}$ & $6.94 \mathrm{~d}$ & $5.77 \mathrm{de}$ \\
\hline Acifluorfen $^{c}$ & 300 & $70 \mathrm{c}$ & $67 \mathrm{bc}$ & $5.96 \mathrm{~cd}$ & $3.99 \mathrm{cde}$ \\
\hline Acifluorfen & 600 & $83 \mathrm{abc}$ & $59 c$ & $6.03 \mathrm{~cd}$ & 3.14 bcde \\
\hline Bentazon $^{c}$ & 1080 & $98 \mathrm{a}$ & $97 \mathrm{ab}$ & $0.45 \mathrm{ab}$ & $0.15 \mathrm{ab}$ \\
\hline Bentazon Forte & 1080 & $98 \mathrm{a}$ & $97 \mathrm{ab}$ & $0.81 \mathrm{ab}$ & $0.21 \mathrm{abc}$ \\
\hline Bentazon/acifluorfen ${ }^{\mathrm{d}}$ & 840 & $88 \mathrm{abc}$ & $78 \mathrm{abc}$ & $2.21 \mathrm{abc}$ & 1.84 abcde \\
\hline Bentazon + fomesafen ${ }^{e}$ & $840+140$ & $96 \mathrm{ab}$ & $91 \mathrm{abc}$ & $1.94 \mathrm{abc}$ & $0.94 \mathrm{abcd}$ \\
\hline Bentazon + fomesafen ${ }^{f}$ & $840+140$ & $97 a$ & $99 a$ & $0.48 \mathrm{ab}$ & $0.19 \mathrm{ab}$ \\
\hline Bentazon Forte + fomesafen & $1080+140$ & $98 \mathrm{a}$ & $96 \mathrm{abc}$ & $0.76 \mathrm{ab}$ & $0.12 \mathrm{ab}$ \\
\hline
\end{tabular}

aAbbreviations: POST, postemergence; WAT, weeks after herbicide treatment; ${ }^{\mathrm{b}}$ Includes Turbocharge at $0.5 \%$ $\mathrm{v} / \mathrm{v}$; Includes Assist at $0.5 \% \mathrm{v} / \mathrm{v}$; Includes Assist at $1.5 \mathrm{l} \cdot \mathrm{ha}^{-1}$; ${ }^{\mathrm{e}}$ Includes Assist at $2.0 \mathrm{l} \cdot \mathrm{ha}^{-1}$; Includes Turbocharge at $0.51 \cdot \mathrm{ha}^{-1}$.

Table 6. Percent visible control 4 and 8 WAT, density and dry weight of wild mustard after various herbicides were applied POST at Exeter, ON, Canada (2017-2019). Means followed by a different letter within a column are significantly different according to a Tukey-Kramer multiple range test at $\mathrm{P}<0.05$. $^{\mathrm{a}}$

\begin{tabular}{|c|c|c|c|c|c|}
\hline \multirow[t]{2}{*}{ Treatment } & \multirow{2}{*}{$\frac{\text { Rate }}{\left(\mathrm{g}_{\mathrm{ai} \mathrm{ha}}{ }^{-1}\right)}$} & \multicolumn{2}{|c|}{ Control (\%) } & \multirow[b]{2}{*}{$\begin{array}{c}\text { Density } \\
\left(\text { no. } \mathrm{m}^{-2}\right)\end{array}$} & \multirow[b]{2}{*}{$\begin{array}{c}\text { Dry weight } \\
\left(\mathrm{g} \cdot \mathrm{m}^{-2}\right)\end{array}$} \\
\hline & & $4 \mathrm{WAT}$ & $8 \mathrm{WAT}$ & & \\
\hline Weed-free control & & 100 & 100 & $0.0 \mathrm{a}$ & $0.0 \mathrm{a}$ \\
\hline Weedy control & & $0 \mathrm{~b}$ & $0 \mathrm{~b}$ & $138.8 \mathrm{~b}$ & $186.2 \mathrm{~b}$ \\
\hline Fomesafen $^{\mathrm{b}}$ & 240 & $100 \mathrm{a}$ & $100 \mathrm{a}$ & $0.0 \mathrm{a}$ & $0.0 \mathrm{a}$ \\
\hline Acifluorfen $^{c}$ & 300 & $99 \mathrm{a}$ & $100 \mathrm{a}$ & $0.0 \mathrm{a}$ & $0.0 \mathrm{a}$ \\
\hline Acifluorfen & 600 & $100 \mathrm{a}$ & $100 \mathrm{a}$ & $0.0 \mathrm{a}$ & $0.0 \mathrm{a}$ \\
\hline Bentazon $^{c}$ & 1080 & $100 \mathrm{a}$ & $100 \mathrm{a}$ & $0.2 \mathrm{a}$ & $0.1 \mathrm{a}$ \\
\hline Bentazon Forte & 1080 & 99 a & $100 \mathrm{a}$ & $0.3 \mathrm{a}$ & $0.1 \mathrm{a}$ \\
\hline Bentazon/acifluorfen ${ }^{\mathrm{d}}$ & 840 & $100 \mathrm{a}$ & $100 \mathrm{a}$ & $0.0 \mathrm{a}$ & $0.0 \mathrm{a}$ \\
\hline Bentazon + fomesafen ${ }^{e}$ & $840+140$ & $100 \mathrm{a}$ & $100 \mathrm{a}$ & $0.1 \mathrm{a}$ & $0.0 \mathrm{a}$ \\
\hline Bentazon + fomesafen $^{\mathrm{f}}$ & $840+140$ & $100 \mathrm{a}$ & $100 \mathrm{a}$ & $0.0 \mathrm{a}$ & $0.0 \mathrm{a}$ \\
\hline Bentazon Forte + fomesafen & $1080+140$ & $100 \mathrm{a}$ & $100 \mathrm{a}$ & $0.0 \mathrm{a}$ & $0.0 \mathrm{a}$ \\
\hline
\end{tabular}

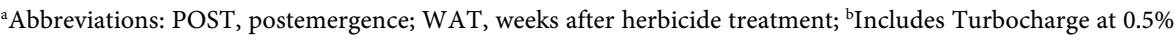
$\mathrm{v} / \mathrm{v}$; Includes Assist at $0.5 \% \mathrm{v} / \mathrm{v}$; ${ }^{\mathrm{d}}$ Includes Assist at $1.51 \cdot \mathrm{ha}^{-1}$; ${ }^{\mathrm{e}}$ Includes Assist at $2.0 \mathrm{l} \cdot \mathrm{ha}^{-1}$; Includes Turbocharge at $0.5 \mathrm{l} \cdot \mathrm{ha}^{-1}$. 


\subsubsection{Bentazon}

At 4 and 8 WAT, bentazon or bentazon Forte applied POST at $1080 \mathrm{~g}$ ai ha ${ }^{-1}$ controlled A. retroflexus $46 \%$ to $64 \%$, A. artemesiifolia $51 \%$ to $61 \%, C$. album $55 \%$ to $87 \%$, $H$. trionum $97 \%$ to $98 \%$, and $S$. arvensis $99 \%$ to $100 \%$ (Tables 2-6). There was generally no difference between bentazon and bentazon Forte for the control of weeds evaluated except for $C$. album which was controlled more effectively with bentazon Forte ( $78 \%$ to $87 \%$ ) compared to bentazon (55\% to $66 \%$ ). Bentazon or bentazon Forte applied POST at $1080 \mathrm{~g}^{\text {ai }} \mathrm{ha}^{-1}$ did not reduce density or dry weight of $A$. retroflexus and $A$. artemesiifolia, but decreased density and dry weight of $H$. trionum up to $98 \%$ and $S$. arvensis $100 \%$ (Tables 2-6). Bentazon did not reduce density and dry weight of $C$. album, but bentazon Forte decreased density and dry weight of $C$. album $67 \%$ and $66 \%$, respectively. In other studies, bentazon Forte applied POST at $840 \mathrm{~g}$ ai ha $^{-1}$ controlled $A$. retroflexus $85 \%$ to $86 \%$, A. artemesiifolia $63 \%$ to $66 \%$, C. album $88 \%$ to $91 \%$ and S. arvensis $96 \%$ to $97 \%[14]$.

\subsubsection{Bentazon/Acifluorfen}

At 4 and 8 WAT, bentazon/acifluorfen (with Assist ${ }^{\circ}$ ) applied POST at $840 \mathrm{~g}$ ai ha $^{-1}$ controlled $A$. retroflexus $46 \%$ to $67 \%$, A. artemesiifolia $59 \%$ to $66 \%, C$. album $59 \%$ to $72 \%, H$. trionum $78 \%$ to $88 \%$, and $S$. arvensis $100 \%$ (Tables 2-6). Bentazon/acifluorfen (with Assist ${ }^{\circ}$ ) applied POST at $840 \mathrm{~g}$ ai ha ${ }^{-1}$ did not reduce density or dry weight of $A$. retroflexus, $A$. artemesiifolia and $C$. album, but decreased density and dry weight of $H$. trionum as much as $84 \%$ and $S$. arvensis $100 \%$ (Tables 2-6).

\subsubsection{Bentazon + Fomesafen}

Bentazon + fomesafen (with Assist $)$, bentazon + fomesafen (with Turbocharge $\left.{ }^{\circ}\right)$ applied POST at $840+140 \mathrm{~g}$ ai ha ${ }^{-1}$ controlled A. retroflexus $43 \%$ to $70 \%, A$. artemesiifolia $63 \%$ to $72 \%, C$. album $52 \%$ to $83 \%, H$. trionum $91 \%$ to $99 \%$, and $S$. arvensis $100 \%$ (Tables 2-6). There were generally no statistical differences between bentazon + fomesafen (with Assist) ) and bentazon + fomesafen (with Turbocharge) for the control of weeds evaluated. Bentazon + fomesafen (with Assist $^{\circ}$ ) and bentazon + fomesafen (with Turbocharge) applied POST at $840+$ $140 \mathrm{~g}$ ai ha ${ }^{-1}$ did not reduce density or dry weight of $A$. artemesiifolia, but decreased density of $A$. retroflexus and C. album $54 \%$ and $68 \%$, respectively. These herbicides also reduced $H$. trionum density $86 \%$ to $96 \%$ and dry weight $89 \%$ to $98 \%$. The density and dry weight of $S$. arvensis was reduced by $100 \%$ compared to the weedy control (Tables 2-6). In other studies, bentazon + fomesafen applied POST provided $77 \%$ to $87 \%$ control of $C$. album in dry bean [16].

\subsubsection{Bentazon Forte + Fomesafen}

At 4 and 8 WAT, bentazon Forte + fomesafen applied POST at $1080+140 \mathrm{~g}$ ai ha $^{-1}$ controlled $A$. retroflexus $36 \%$ to $68 \%$, A. artemesiifolia $69 \%$ to $70 \%, C$. album $65 \%$ to $82 \%, H$. trionum 96 to $98 \%$, and $S$. arvensis $100 \%$ (Tables 2-6). Bentazon Forte + fomesafen applied POST at $1080+140 \mathrm{~g}_{\text {ai ha }}{ }^{-1}$ did not reduce 
density or dry weight of $A$. retroflexus and $A$. artemesiifolia, but reduced $C$. album, $H$. trionum and $S$. arvensis density $64 \%, 94 \%$ and $100 \%$ and dry weight $63 \%, 99 \%$ and $100 \%$, respectively (Tables $2-6$ ). In other studies, bentazon Forte + fomesafen applied POST at $840+140 \mathrm{~g}$ ai ha ${ }^{-1}$ controlled $A$. retroflexus $84 \%$ to $86 \%$, A. artemesiifolia $91 \%$ to $93 \%$ and C. album $84 \%$ to $87 \%$ [14].

\section{Conclusion}

This study concludes that fomesafen, acifluorfen (with Assist ${ }^{\circ}$ ) and acifluorfen (without adjuvants) cause up to 6\% crop injury and weed interference with these herbicides reduced azuki bean yield up to $42 \%$. Additionally, bentazon, bentazon Forte, bentazon/acifluorfen, bentazon + fomesafen (with Assist'), bentazon + fomesafen (with Turbocharge ${ }^{\circ}$ ) and bentazon Forte + fomesafen can cause up to $16 \%$ crop injury and weed interference with these herbicides can reduce azuki bean yield up to $53 \%$. Fomesafen and acifluorfen (with/without Assist) did not affect maturity, but bentazon, bentazon Forte, bentazon/acifluorfen, bentazon + fomesafen (with Assist $)^{\circ}$ ), bentazon + fomesafen (with Turbocharge ${ }^{\circ}$ ) and bentazon Forte + fomesafen delayed maturity of azuki bean (Table 1 ). Weed interference reduced azuki bean yield $74 \%$ compared to the weed-free control. Fomesafen, acifluorfen (with Assist'), acifluorfen, bentazon, bentazon Forte, bentazon/acifluorfen, bentazon + fomesafen (with Assist'), bentazon + fomesafen (with Turbocharge $)$ and bentazon Forte + fomesafen generally provided inadequate control of $A$. retroflexus, $A$. artemesiifolia and $C$. album. Fomesafen, acifluorfen (with Assist') and acifluorfen (without adjuvants) did not adequately control of $H$. trionum, but bentazon, bentazon Forte, bentazon/acifluorfen, bentazon + fomesafen (with Assist ${ }^{\circ}$ ), bentazon + fomesafen (with Turbocharge ${ }^{\circ}$ ) and bentazon Forte + fomesafen provided good to excellent control of $H$. trionum. All herbicides evaluated provide excellent control of $S$. arvensis in azuki bean. Results indicate that all herbicide programs that include bentazon do not have an adequate margin of crop safety for weed management in azuki bean in Ontario.

\section{Acknowledgements}

Funding for this project was provided in part by the Ontario Bean Growers (OBG).

\section{Conflicts of Interest}

The authors declare no conflicts of interest regarding the publication of this paper.

\section{References}

[1] Statista (2020) Production Volume of Dry Beans in Canada from 2016/17 to 2019/20.

https://www.statista.com/statistics/819203/production-volume-of-dry-beans-canada

[2] OMAFRA (2020) 2019 Dry Edible Bean Summary. 
http://www.omafra.gov.on.ca/english/crops/field/reports/2019summary-ediblebean. $\underline{\mathrm{htm}}$

[3] Lumpkin, T.A. and McClary, D.C. (1994) Azuki Bean: Botany, Production and Uses. CAB International, Wallingford.

[4] McClary, D.C., Hang, A.N., Gilliland, G.C., Babcock, J.M., Lumpkin, T.A., Ogg, A.G. and Tanigoshi, L.K. (1993) Herbicides for Azuki Production. In: Janick, J. and Simon, J.E., Eds., New Crops, Wiley, New York, 590-594.

[5] Soltani, N., Dille, J.A., Burke, I.C., Everman, W.J., VanGessel, M.J., Davis, V.M. and Sikkema, P.H. (2016) Potential Corn Yield Losses from Weeds in North America. Weed Technology, 30, 979-984. https://doi.org/10.1614/WT-D-16-00046.1

[6] Soltani, N., Dille, J.A., Burke, I.C., Everman, W.J., VanGessel, M.J., Davis, V.M. and Sikkema, P.H. (2017) Perspectives on Potential Soybean Yield Losses from Weeds in North America. Weed Technology, 31, 148-154. https://doi.org/10.1017/wet.2016.2

[7] Soltani, N., Dille, J.A., Burke, I.C., Everman, W.J., VanGessel, M.J., Davis, V.M. and Sikkema, P.H. (2018) Potential Yield Loss in Dry Bean Crops Due to Weeds in the United States and Canada. Weed Technology, 32, 342-346.

https://doi.org/10.1017/wet.2017.116

[8] Ontario Ministry of Agriculture and Food and Rural Affairs (OMAFRA) (2019) Guide to Weed Control, Publication 75. Toronto, 1-396.

[9] Shaner, D. (2014) Herbicide Handbook. Tenth Edition, Weed Sci. Soc. Am., Champaign, $458 \mathrm{p}$.

[10] SAS Institute Inc. (2016) Base SAS ${ }^{\varpi} 9.4$ Procedures Guide: Statistical Procedures. Fifth Edition, SAS Institute Inc., Cary.

[11] Stewart, C.L., Nurse, R.E., Gillard, C.L. and Sikkema, P.H. (2010) Tolerance of Adzuki Bean (Vigna angularis) to Preplant-Incorporated, Pre-Emergence and Post-Emergence Herbicides in Ontario, Canada. Weed Biology and Management, 10, 40-47. https://doi.org/10.1111/j.1445-6664.2010.00365.x

[12] Soltani, N., Shropshire, C. and Sikkema, P.H. (2017) Sensitivity of Adzuki Bean (Vigna angularis) to Acifluorfen, Fomesafen, Bentazon, Imazethapyr and Halosulfuron-Methyl Applied Postemergence. American Journal of Plant Sciences, 8, 1092-1099. https://doi.org/10.4236/ajps.2017.85072

[13] Powell, G., Sprague, C. and Renner, K.A. (2004) Adzuki Bean: Weed Control and Production Issues. North Central Weed Science Proceedings, Vol. 59, 32.

[14] Soltani, N., Nurse, R.E. and Sikkema, P.H. (2013) Weed Management in White Beans with Postemergence Herbicide Tankmixes. Canadian Journal of Plant Science, 93, 669-674. https://doi.org/10.4141/cjps2012-273

[15] Bailey, W.A., Wilson, H.P. and Hines, T.E. (2003) Weed Control and Snap Bean (Phaseolus vulgaris) Response to Reduced Rates of Fomesafen. Weed Technology, 17, 269-275. https://doi.org/10.1614/0890-037X(2003)017[0269:WCASBP]2.0.CO;2

[16] Wilson, R.G. (2005) Response of Dry Bean and Weeds to Fomesafen and Fomesafen Tank Mixtures. Weed Technology, 19, 201-206.

https://doi.org/10.1614/WT-04-166R 